\title{
Provably Correct Runtime Enforcement of Non-interference Properties ${ }^{\star}$
}

\author{
V.N. Venkatakrishnan ${ }^{1}$, Wei Xu ${ }^{2}$, Daniel C. DuVarney ${ }^{2}$, and R. Sekar ${ }^{2}$ \\ ${ }^{1}$ Department of Computer Science, University of Illinois at Chicago, \\ Chicago, IL 60607 \\ venkat@cs.uic.edu \\ 2 Department of Computer Science, Stony Brook University, \\ Stony Brook, NY 11794-4400 \\ \{weixu, dand, sekar\}@cs.sunysb.edu
}

\begin{abstract}
Non-interference has become the standard criterion for ensuring confidentiality of sensitive data in the information flow literature. However, application of non-interference to practical software systems has been limited. This is partly due to the imprecision that is inherent in static analyses that have formed the basis of previous non-interference based techniques. Runtime approaches can be significantly more accurate than static analysis, and have often been more successful in practice. However, they can only reason about explicit information flows that take place via assignments in a program. Implicit flows that take place without involving assignments, and can be inferred from the structure and/or semantics of the program, are missed by runtime techniques. This paper seeks to bridge the gap between the accuracy provided by runtime techniques and the completeness provided by static analysis techniques. In particular, we develop a hybrid technique that relies primarily on runtime information-flow tracking, but augments it with static analysis to reason about implicit flows that arise due to unexecuted paths in a program. We prove that the resulting technique preserves non-interference, while providing some of the traditional benefits of dynamic analysis such as improved accuracy.
\end{abstract}

\section{Introduction}

Protecting the privacy of personal information has become one of the main challenges facing the Internet today. Although traditional access control mechanisms can prevent information from being given to unauthorized principals, they don't address the central problem in privacy, namely, information flow control. The domain of information flow control begins at the point where sensitive information is handed to a piece of software, and governs the manner in which this software uses this information. The primary concern is whether sensitive information may

\footnotetext{
* This research was supported in part by NSF grants CCR-0098154, CCR-0208877 and CNS-0551660, and an ONR grant N000140110967.
} 
flow into (or influence) data that may be read by unauthorized principals. In addition to privacy, information flow techniques can also address integrity concerns. The term "taint" is used in place of "information flow" in the context of integrity, and the techniques are concerned with ensuring that untrustworthy data does not influence data whose trustworthiness needs to be preserved.

There are two basic approaches for dealing with information flows in a program: static analysis and runtime-tracking. A static analysis technique has an advantage over runtime-tracking in terms of runtime performance. However, static analysis techniques need to reason about all possible executions of a program, and reject programs that can potentially leak sensitive information in some runs. In particular, the following weaknesses of static analysis techniques make it significantly less accurate than runtime techniques:

- Approximations needed to ensure termination of static analysis. Like most problems in program analysis, it is in general undecidable whether a program contains a prohibited information flow. Approximations need to be made in order to produce a decidable procedure, and these approximation will negatively impact accuracy. In contrast, runtime techniques concern themselves only with the execution path that was actually taken at runtime, and hence can remain decidable without having to resort to approximations.

- Inability to support programs that occasionally leak information. Consider a program that sends a crash report to its developer, and assume that this report contains sensitive information. Under normal conditions, there may not be any leak. Such a program can be supported by a runtime-tracking technique by simply suppressing unacceptable flows at runtime, i.e., by disallowing the crash report from being sent. A purely static analysis technique would have to altogether disallow any use of such programs.

- Inability to support applications where data sensitivity is dynamically determined. Consider a web browser that interacts with a number of web sites, some of which handle sensitive data and the others don't. Thus, the sensitivity of a piece of information received on a communication channel is determined at runtime, based on the identity of the web site providing the information. Naturally, purely static analysis techniques require sensitivity information to be specified statically, and hence have a hard time coping with such applications.

In fact, these precision issues are sufficiently problematic in the related domain of access control so that static analysis is seldom used in these systems. Within the domain of information flow, runtime techniques [72622834] have also enjoyed more practical applications as compared to their static analysis counterparts.

In spite of these precision issues, static analysis has been the predominant technique in recent information flow literature 2023231 . This is because runtime techniques aren't able to capture so-called implicit flows that do not involve explicit assignments, but can be inferred from the structure of the program. For instance, consider the following program, where $\mathrm{h}$ contains sensitive data but 1 should not, since the latter is being printed to a public channel. 


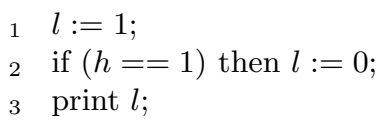

Assume that $\mathrm{h}$ take only two values: 0 or 1 . In this case, the value of $\mathrm{h}$ can be determined from the value of 1 printed by the program. However, a runtime tracking technique will not be able to infer this dependency, since there were no explicit actions that transfer information from $\mathrm{h}$ to 1 . Consequently, runtime techniques are incomplete with respect to the notion of noninterference [14, which formed the basis of all the above static information flow analysis techniques. Noninterference states that the public outputs of a program should not be changed in any way by changes to its sensitive inputs.

It has often been stated that runtime techniques cannot be expected to preserve noninterference since the presence of information flow is dependent on program paths not taken during an execution [24]. It seems natural to conclude that runtime techniques, which make their decisions based on the actual execution trace taken at runtime, cannot detect such flows. We present a result in this paper that, on the surface, seems to contradict this "conventional wisdom." In particular, we present a runtime information-flow tracking technique that preserves non-interference. Our technique first employs a program transformation approach to encode information flows that take place due to unexecuted paths into other paths that are, in fact, executed. This transformation itself is guided by a static analysis. This transformation contains additional instructions in the program that perform runtime informationflow tracking. In this paper, we present the rules for such a transformation and formally establish that this transformation technique preserves non-interference, i.e., it ensures that no information about the sensitive variables used by a program can be inferred from public outputs of the program.

The nature of programs handled by this approach are those that are singlethreaded and deterministic. In this work, we do not address information leaks resulting from timing, storage and termination channels [24]. The rest of the paper is organized as follows. In Section 2, the details of the analysis and transformation are presented, including a small example of its use. In Section 3 the proof of correctness with respect to the non-interference property is presented. Section 4 discusses practical issues in adoption of the runtime enforcement technique. Section 5 discusses related work. Finally, Section 6 provides a summary of our results.

\section{The Transformation}

The Language. We use a simple imperative language with procedures for our discussion. The syntax of expressions and statements in the language appear in Figure 1 The language has basic arithmetic and logical expressions (composed using binary operators ( $b o p)$ and unary operators (uop)), assignments, conditionals, loops, procedure call, sequencing and skip statements. For procedures, a call-by-value semantics is assumed. Otherwise, the semantics of this language 


$$
\begin{gathered}
\text { [expr] } \\
\text { [stmt] } S:=c|x| e_{1} \text { bop } e_{2} \mid \text { uop } e_{1} \\
\text { call } f(e)\left|S_{1} ; S_{2}\right| \text { skip }
\end{gathered}
$$

Fig. 1. The syntax of expressions and statements in the language

is standard. Also, we focus on a simple presentation where the results of the transformation are un-optimized; optimizations are discussed later.

The variables in the language can have security types high and low. For instance, a high security variable can represent a sensitive channel from which sensitive information is read by the program. A low security variable can represent a public input or output channel (such as an insecure network read or a print statement), and hence any assignment of sensitive information to these variables constitutes illegal flow of information. For the variables that are not assigned with high or low security types based on the security policies, they represent intermediate variables and can hold values from high or low security variables because they are not publicly visible. Constants are always of type low.

Basic Idea. In our approach, information flow is tracked through the use of label variables. The idea is simple: for each variable in the program, a new boolean label variable is created. At runtime, this label variable records the flow of sensitive information into this variable. When execution reaches an assignment statement that assigns to a low security variable, the corresponding label variable is examined for the presence of sensitive data. If such sensitive data is indeed present, the execution of the program is terminated.

For any variable $x$, let $\mathcal{L}(x)$ denote the label variable that tracks flows to $x$ at any program point. The label variable is a boolean variable and the value stored in a label variable reflects whether a variable contains sensitive information (in which case it is 1 ), or not. A label value that is 0 denotes the absence of sensitive information. For example, the execution of the statement $x:=y+z$ results in updating $\mathcal{L}(x)$ to $\mathcal{L}(y) \vee \mathcal{L}(z)$. Furthermore, if either $y$ or $z$ contain sensitive data read from a high security variable, then their corresponding label variable $(\mathcal{L}(y)$ or $\mathcal{L}(z))$ is set to true, and consequently, $\mathcal{L}(x)$ will be true after the assignment. In addition, to simplify the notation, for any expression $e$, we also use $\mathcal{L}(e)$ to denote the label value obtained by the disjunction of all the label variables corresponding to variables appearing in the expression $e$. So, for the expression $x * z+y, \mathcal{L}(x * z+y)$ is $\mathcal{L}(x) \vee \mathcal{L}(z) \vee \mathcal{L}(y)$.

The Analysis. The simple idea illustrated above would work only for explicit flows through assignments. On entering a conditional branch, there is an implicit flow of information from the conditional to all the variables assigned in both the branches. To compute this set of variables a static analysis is used. The results of this analysis (described below) are present in an environment, denoted by $\Psi$. The transformation then makes use of the results of this analysis. (Our transformation mechanism can make use of a variety of analysis mechanisms, and hence here 


$$
\begin{aligned}
& {\left[\text { ASSIGN1] } \frac{\Gamma \vdash x: \tau \text { where } \tau \neq \text { low }}{x:=e \rightarrow \Gamma, \Psi \mathcal{L}(x):=\mathcal{L}(e) \vee \mathcal{L}(p c) ;}\right.} \\
& x:=e \\
& {\left[\text { ASSIGN2] } \frac{\Gamma \vdash x: \tau \text { where } \tau=\text { low }}{x:=e \rightarrow_{\Gamma, \Psi} \mathcal{L}(x):=\mathcal{L}(e) \vee \mathcal{L}(p c) ;}\right.} \\
& \text { call policy_check }(\mathcal{L}(x)) \text {; } \\
& x:=e \\
& {[\mathrm{IF}] \frac{\Psi \vdash x_{1}, x_{2}, \ldots, x_{n} \in U p d\left(S_{1}\right) \cup U p d\left(S_{2}\right) \quad S_{1} \rightarrow_{\Gamma, \Psi} S_{1}^{*} \quad S_{2} \rightarrow_{\Gamma, \Psi} S_{2}^{*}}{\text { if } e \text { then } S_{1} \text { else } S_{2} \text { endif } \rightarrow_{\Gamma, \Psi} \mathcal{L}\left(p c_{1}\right):=\mathcal{L}(p c) ;}} \\
& \mathcal{L}(p c):=\mathcal{L}(p c) \vee \mathcal{L}(e) \\
& \text { if } e \text { then } S_{1}^{*} \text { else } S_{2}^{*} \text { endif; } \\
& \mathcal{L}\left(x_{i}\right):=\mathcal{L}\left(x_{i}\right) \vee \mathcal{L}(p c) ;(1 \leq i \leq n) \\
& \mathcal{L}(p c):=\mathcal{L}\left(p c_{1}\right) \\
& \text { [WHILE] } \frac{\Psi \vdash x_{1}, x_{2}, \ldots, x_{n} \in U p d(S) \quad S \rightarrow_{\Gamma, \Psi} S^{*}}{\text { while } e \text { do } S \text { done } \rightarrow \Gamma, \Psi \mathcal{L}\left(p c_{1}\right):=\mathcal{L}(p c) ;} \\
& \mathcal{L}(p c):=\mathcal{L}(p c) \vee \mathcal{L}(e) \\
& \text { while } e \text { do } \\
& S^{*} \text {; } \\
& \mathcal{L}(p c):=\mathcal{L}(p c) \vee \mathcal{L}(e) ; \\
& \text { done; } \\
& \mathcal{L}\left(x_{i}\right):=\mathcal{L}\left(x_{i}\right) \vee \mathcal{L}(p c) ;(1 \leq i \leq n) \\
& \mathcal{L}(p c):=\mathcal{L}\left(p c_{1}\right) \\
& {[\mathrm{CALL}] \frac{x_{1} \text { is a fresh temporary variable }}{\text { call } f(e) \rightarrow \Gamma, \Psi} \begin{array}{c}
\mathcal{L}\left(x_{1}\right):=\mathcal{L}(e) \vee \mathcal{L}(p c) ; \\
\text { call } f\left(e, \mathcal{L}\left(x_{1}\right)\right)
\end{array}} \\
& {[\mathrm{SEQ}] \frac{S_{1} \rightarrow_{\Gamma, \Psi} S_{1}^{*} \quad S_{2} \rightarrow_{\Gamma, \Psi} S_{2}^{*}}{S_{1} ; S_{2} \rightarrow_{\Gamma, \Psi} S_{1}^{*} ; S_{2}^{*}}} \\
& {[\mathrm{SKIP}] \overline{\text { skip } \rightarrow \Gamma, \Psi \text { skip }}}
\end{aligned}
$$

Fig. 2. Transformation $\rightarrow_{\Gamma, \Psi}$, parameterized by $\Gamma$ (initial type environment that encodes the security policy) and $\Psi$ (results of the static analysis that are used to account for implicit flows)

we only discuss the results that are required from the static analysis; we discuss possible strategies for performing these analysis in a later section.)

The analysis computes the following: If $S$ denotes a (possibly compound) statement, the set $U p d(S)$ denotes all the variables that get assigned in the statement $S$. The analysis computes a conservative upper bound in case a precise estimation of the set of variables that are updated is not possible.

Using the results of the analysis, the program is transformed such that, on entering a conditional branch, the value of the label variable corresponding to the 
condition expression (associated with enclosing nested conditionals), is stored in a global variable called $\mathcal{L}(p c)$ that is used to track implicit flows. For all the assignments that happen inside both branches, the label variables of the lefthand side expressions are updated with the value of the implicit flows. This is done by a disjunction with $\mathcal{L}(p c)$. Thus, $\mathcal{L}(p c)$ includes the combined effect of the enclosing conditionals and loops. When a conditional branch is exited, the value of $\mathcal{L}(p c)$ is restored to its previous value that existed before entering the branch.

The type environment $\Gamma$ represents the initial type assignment of high and low security variables. This environment is directly initialized from the security policy. For all the variables that are typed high the label variables are initialized to true. All the other label variables are initialized to false. When a procedure is invoked, the local variables are initialized in a similar fashion.

The transformation. Figure 2 presents the transformation rules in an inference style formalism. The transformation is denoted by the symbol $\rightarrow_{\Gamma, \Psi}$, as it takes as inputs the security-type environment $\Gamma$ and the results from the static analysis $\Psi$.

The transformation rules update the label variables to reflect the sensitivity of the information that is contained in the corresponding program variables. In the transformation rules, updates to the label variables according to the kind of the statement are shown. These updates are present in the transformed program and are executed at run-time. We point out that the policy_check procedure checks whether the current assignment (to be performed) is legal. It does so by checking the sensitivity of the data being assigned to a low security variable (by checking whether the boolean value corresponding to its argument is true). If so, then the execution is terminated before the actual information leak occurs. This procedure may be defined as follows:

$$
\text { policy_check }(\operatorname{var} l a b)\{\text { if }(l a b) \text { then halt else skip; }\}
$$

Below, we briefly explain the transformation rules.

The transformation rule SKIP for the skip statement is obvious. The rule SEQ for the sequential composition statement of statements $S_{1}$ and $S_{2}$ defines that the individual statements are transformed to $S_{1}^{*}$ and $S_{2}^{*}$ respectively.

ASSIGN1 involves assignment to a non-low security variable (which is not observable). For this rule, the effect of all label variables of the expression is computed and assigned to the label variable for the variable on the LHS. The label variable $\mathcal{L}(p c)$ is used to account for implicit flows.

For the assignment rule ASSIGN2, the LHS is a low security variable. In addition to performing actions similar to ASSIGN1, care should be exercised to check whether there is an information leak from the expression in the RHS. For this purpose, the procedure policy_check is called, which checks the value of the label of the RHS. If it is true, then there is a potential information leak, and the program is halted. 
The transformation rule IF for the if-then-else statement is more complicated. We explain each of the five steps in this transformation. First, the current value of $\mathcal{L}(p c)$ is recorded in a fresh variable $\mathcal{L}\left(p c_{1}\right)$ (for recovering and restoring it after this statement). Secondly, the value of $\mathcal{L}(p c)$ is updated with the value of the label variables of the variables involved in the condition. This is done to account for implicit flows to any assignments to the body of the then or else branches. In the third step, the transformation is applied further to both branches of the statement. This is done by transforming the individual statements $S_{1}$ and $S_{2}$ to $S_{1}^{*}$ and $S_{2}^{*}$ respectively. In the fourth step, the transformation accounts for implicit flows through assignments in both branches. This is done by consulting the results of the analysis for the update sets of statements $S_{1}$ and $S_{2}$. The union of these sets, represented by $\operatorname{Upd}\left(S_{1}\right) \cup U p d\left(S_{2}\right)$, denotes the set of variables that will carry information about the condition expression as a result of implicit flows. We update their label variables with the current value of $\mathcal{L}(p c)$ to account for implicit flows. (The transformation does this for all the variables in the set, $\operatorname{Upd}\left(S_{1}\right) \cup \operatorname{Upd}\left(S_{2}\right)$. This is shown in the transform rule for variables $x_{1}, x_{2}, \ldots, x_{n}$ in this set.) Finally, in the fifth step, the value of $\mathcal{L}(p c)$ is restored to the value it originally had before entering the conditional (through the use of $\mathcal{L}\left(p c_{1}\right)$ ).

Notice that the transformation rule WHILE for the while statement is similar to the if-then-else statement. To account for implicit flows into variables from the expression in the condition, the value of $\mathcal{L}(p c)$ is augmented with the value of the expression $e$, before and during each iteration of the loop. (The loop may modify the variables in the expression, and therefore the expression's label value may change). The labels of variables assigned in the loop (such as $x_{i}$ ), are updated after the loop to account for implicit flows from $e$. (Inside the body, the labels of such variables are updated in the corresponding assignment statements). The body of the while statement is then transformed. Note that $\mathcal{L}(p c)$ is restored after the execution of the while statement to its original value. (Note that, for an external observer who could observe the timing/termination effects of programs, this could leak one bit of information about the condition. However, we do not address timing/termination channels in this work.)

As shown in the transformation rule CALL, a call statement is transformed as follows: A fresh label variable corresponding to the call argument expression is created. Then the flows for each of the individual variables in the expression is accounted for through $\mathcal{L}(e)$. Finally, a call to the transformed procedure (which is explained below) is made with this additional argument.

The transformation of procedure definitions is done in a similar fashion (not shown in figure). The procedure is modified to include an additional label variable for the argument to the procedure. Further, label variables corresponding to local variables are created. The statement body is then transformed according to the above mentioned rules.

Figure 3 (a) \& (b) show a program fragment and its transformed version respectively. In the transformed program, a label variable $\mathcal{L}(x)$ is denoted by $x^{\prime}$. 


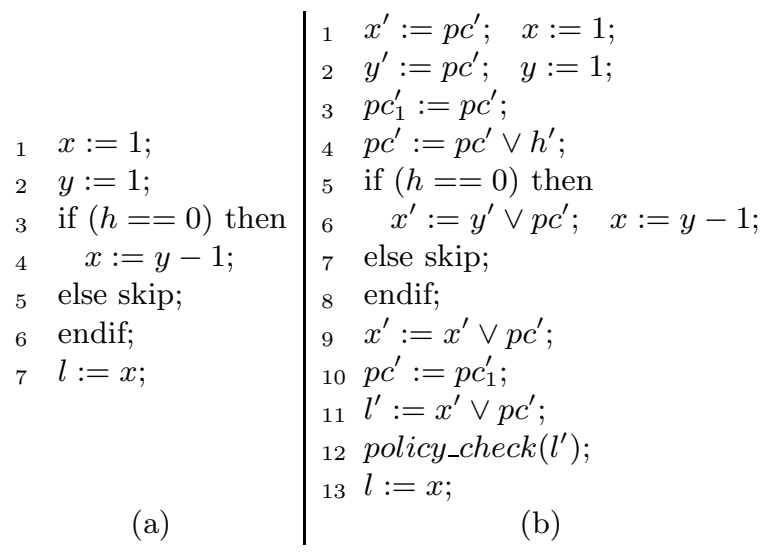

Fig. 3. (a) Example program and (b) its transformation

\section{Formalization of Correctness Criteria}

First, we claim the simple result that the transformation is semantics preserving, providing that the call to policy_check does not halt. This is clear as the transformation only makes updates to the label variables (such as $\mathcal{L}(x)$ and $\mathcal{L}(p c)$ ). The only construct in our transformation that has the ability to alter the execution is policy_check. Specifically, policy_check acts as a halt instruction if the condition check succeeds, and acts as a skip instruction if the check fails. All the additional instructions in our transformation only make updates to label variables. Hence, the following theorem is true.

Theorem 1 (Semantics preservation). Let $P$ be any program, and $P^{*}$ the transformed program obtained applying the transformation $\rightarrow_{\Gamma, \Psi}$ on $P$. Then, $P$ and $P^{*}$ are semantically equivalent on all executions in which policy_check never halts $P^{*}$.

Next, we show the correctness of the transformation with respect to the noninterference property, which ensures that an attacker cannot learn confidential information by observing only low security variables.

Definition 1 (Non-interference). A program is said to be non-interfering if for any two sets of input values that differ only on their high security values, their corresponding low security output values are the same.

To effectively compare two execution traces, the simple notion of program counter (source line number) is not sufficient as the length of the traces will differ due to the effect of loops and branches. To illustrate this point, consider the program fragment given below (assuming it is defined in a procedure named foo). Let $\mathrm{h}=1$ in the first trace, and $h=2$ in the second. Then, at program location 3 , we cannot compare the trace elements obtained in the first iteration (when $\mathrm{h}=1$ in the first trace), and second iteration in the second trace (when $\mathrm{h}=1$ in the second trace). 


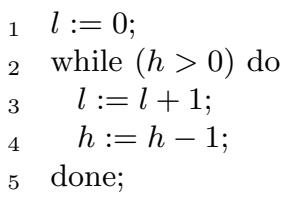

By the following definitions, we factor out the effect of loops by including the loop counter in the value of the label variable of the program location.

Definition 2. A program counter is defined as a finite sequence $\left\langle\right.$ pname, $\ell_{0}$ : $\left.\left\langle\ell_{1}, i_{1}\right\rangle:\left\langle\ell_{2}, i_{2}\right\rangle: \ldots:\left\langle\ell n, i_{n}\right\rangle\right\rangle$.

- pname is the name of the procedure that is currently being executed.

- $\ell_{0}$ is the location (source line number) of the next statement to be executed.

- For all $k$ such that $1 \leq k \leq n, \ell_{k}$ is the location of the while statement immediately enclosing $\ell_{k-1}$.

- For the while statement at location $\ell_{k}(1 \leq k \leq n), i_{k}$ is the current iteration number (e.g., 1 the first time through the loop, 2 the second, and so on).

Here, $\ell_{1}$ is the location of the most immediate (i.e., innermost) while statement enclosing the statement at $\ell_{0}$. Note that there may be no enclosing while, in which case the entire program counter value is simply $\left\langle\right.$ pname, $\left.\ell_{0}\right\rangle$.

Definition 3. An extended program counter within a program $P$ is defined as a sequence of program counters.

$\left\langle\right.$ pname $\left.\left._{1}, \ell_{0}^{1}:\left\langle\ell_{1}^{1}, i_{1}^{1}\right\rangle:\left\langle\ell_{2}^{1}, i_{2}^{1}\right\rangle: \ldots:\left\langle\ell^{1}\right\rangle_{m_{1}}, i_{m_{1}}^{1}\right\rangle\right\rangle,\left\langle\right.$ pname $_{2}, \ell_{0}^{2}:\left\langle\ell_{1}^{2}, i_{1}^{2}\right\rangle:$ $\left.\left\langle\ell_{2}^{2}, i_{2}^{2}\right\rangle: \ldots:\left\langle\ell_{m_{2}}^{2}, i_{m_{2}}^{2}\right\rangle\right\rangle, \ldots,\left\langle\right.$ pname $\left._{n}, \ell_{0}^{n}:\left\langle\ell_{1}^{n}, i_{1}^{n}\right\rangle:\left\langle\ell_{2}^{n}, i_{2}^{n}\right\rangle: \ldots:\left\langle\ell_{m_{n}}^{n}, i_{m_{n}}^{n}\right\rangle\right\rangle$

In this definition, $\ell_{0}^{2}$ refers to the location (in procedure pname $_{2}$ ) from which the call to procedure pname $_{1}$ was made. In general, $\ell_{0}^{n}$ refers to the location in

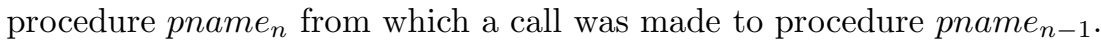

Example: Using the definition of extended program counter, in the first trace, at source line number 3 for the first (and only) iteration of the loop, the extended program counter is $p c_{1}=\langle f o o, 3:(2,1)\rangle$. (program source line 3 , and first iteration of the loop rooted at program line 2$)$. In the second trace, the program location for the first iteration is $p c_{2}=\langle f o o, 3:(2,1)\rangle$ and that for second iteration of the loop is $p c_{3}=\langle f o o, 3:(2,2)\rangle$. Since $p c_{1}$ and $p c_{2}$ are same for the first iterations, the two trace elements can be compared, whereas the $p c_{1}$ and $p c_{3}$ cannot be compared since they belong to different iterations of the loop.

Note that extended program counter values are unique within the same execution (due to the fact that we capture iteration counts and procedure in program counters). Equality of extended program counters is defined as the equality of the individual sequences. A trace itself is obtained by collecting program states at various program points in an execution sequence of a program.

Definition 4. A program state is a pair $\langle p c$, env $\rangle$ where pc is an extended program counter, and env : $(\operatorname{Var} \cup \mathcal{L}(\operatorname{Var}) \cup\{\mathcal{L}(p c)\}) \rightarrow \mathbf{Z}$ is an environment mapping all program variables and label variables to values. 


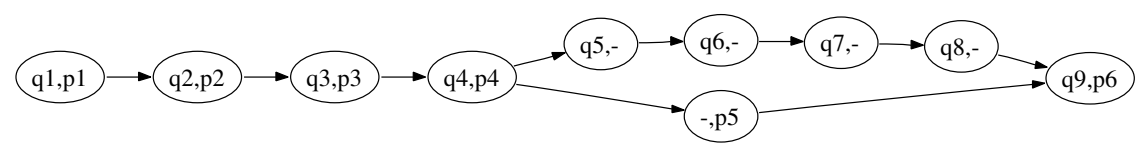

Fig. 4. Example of an execution trace pair

We use the following notation in conjunction with a program state $q$ :

- $p c_{q}$ the extended program counter value of $q$;

- env $v_{q}$ the environment of $q$;

- stmt $_{q}$ the statement at location $p c_{q}$.

Definition 5. A program execution trace is a sequence of program states $q_{0} q_{1} q_{2} \ldots q_{n}$, where $q_{i}$ is the result of executing stmt $q_{q_{i-1}}$ in state $q_{i-1}$. Given two traces $T_{1}=q_{0} q_{1} q_{2} \ldots q_{n}$ and $T_{2}=p_{0} p_{1} p_{2} \ldots p_{m}$ of the same program $P$ with different high security input values, the set of trace pairs, denoted by $\Sigma_{T_{1}, T_{2}}$ is a subset of $T_{1} \times T_{2}$ and is the union of three sets:

$-\left\{\left\langle q_{i}, p_{j}\right\rangle\right\}$ when $p c_{q_{i}}=p c_{p_{j}}$

$-\left\{\left\langle q_{i},-\right\rangle\right\}$ when for all $p \in T_{2}, p c_{q_{i}} \neq p c_{p}$

$-\left\{\left\langle-, p_{j}\right\rangle\right\}$ when for all $q \in T_{1}, p c_{p_{j}} \neq p c_{q}$

Note that, for every $q \in T_{1}$ there is exactly one trace pair involving $q$ and possibly one state from $T_{2}$. A symmetric property holds true for all $p \in T_{2}$.

Definition 6. Given a set $\Sigma_{T_{1}, T_{2}}$ of trace pairs from traces $T_{1}=q_{0} q_{1} q_{2} \ldots q_{n}$ and $T_{2}=p_{0} p_{1} p_{2} \ldots p_{m}$, the stepwise ordering relation $\triangleleft \subseteq \Sigma \times \Sigma$ is defined by the following rules:

- if $\left\langle q_{i}, X\right\rangle \in \Sigma_{T_{1}, T_{2}}$ and $\left\langle q_{i+1}, Y\right\rangle \in \Sigma_{T_{1}, T_{2}}$ then $\left\langle q_{i}, X\right\rangle \triangleleft\left\langle q_{i+1}, Y\right\rangle$ iff $X=$ - or $Y=-$ or $X=p_{j}$ for some $j$ and $Y=p_{j+1}$

- if $\left\langle Z, p_{k}\right\rangle \in \Sigma_{T_{1}, T_{2}}$ and $\left\langle W, p_{k+1}\right\rangle \in \Sigma_{T_{1}, T_{2}}$ then $\left\langle Z, p_{k}\right\rangle \triangleleft\left\langle W, p_{k+1}\right\rangle$ iff $Z=$ - or $W=-$ or $Z=q_{l}$ for some $l$ and $W=q_{l+1}$

By the above definition, the following trace pairs (if they are valid pairs) are related by the stepwise relation: $\left\langle q_{i}, p_{j}\right\rangle \triangleleft\left\langle q_{i+1},-\right\rangle, \quad\left\langle q_{i},-\right\rangle \triangleleft\left\langle q_{i+1},-\right\rangle, \quad\left\langle-, p_{j}\right\rangle \triangleleft$ $\left\langle-, p_{j+1}\right\rangle, \quad\left\langle-, p_{j}\right\rangle \triangleleft\left\langle q_{i}, p_{j+1}\right\rangle$.

Note that for every element $\sigma$ of $\Sigma$, there are at most two successors (corresponding to branch entry points), and at most two predecessors (corresponding to merge points). Also, the structure imposed on $\Sigma$ by $\triangleleft$ is a linear sequence of trace pairs, interrupted by diverging regions where there are exactly two parallel trace pair sequences. At every point (defined by starting with $\sigma_{0}$, and following $\triangleleft)$ there are one or two active trace pairs, up until the point where (possibly) both traces halt. Figure 4 shows a typical trace pair. 
Definition 7. We define the following utility functions/relations:

1. $\operatorname{succ}(\sigma)=\left\{\sigma^{\prime} \in \Sigma \mid \sigma \triangleleft \sigma^{\prime}\right\}$, (successor)

2. $\operatorname{pred}(\sigma)=\left\{\sigma^{\prime} \in \Sigma \mid \sigma^{\prime} \triangleleft \sigma\right\}$, (predecessor)

3. $\triangleleft^{+}=$the transitive closure of $\triangleleft$,

4. $\operatorname{gcai}(i, j)=$ the largest $k$ such that $\sigma_{k} \triangleleft^{*} \sigma_{i}$ and $\sigma_{k} \triangleleft^{*} \sigma_{j}$,

5. $\operatorname{lcdi}(i, j)=$ the least $k$ such that $\sigma_{i} \triangleleft^{*} \sigma_{k}$ and $\sigma_{j} \triangleleft^{*} \sigma_{k}$,

6. $\operatorname{gca}\left(\sigma_{i}, \sigma_{j}\right)=\sigma_{\text {gcai }(i, j)}$ (greatest common ancestor),

7. $\operatorname{lcd}\left(\sigma_{i}, \sigma_{j}\right)=\sigma_{l c d i(i, j)}$ (least common descendant).

8. for any $\Sigma^{\prime} \subset \Sigma_{T_{1}, T_{2}}$, prefix_closed $\left(\Sigma^{\prime}\right)=$ true if a) $\left\langle q_{0}, p_{0}\right\rangle \in \Sigma^{\prime}$, and b) $\forall \sigma \in \Sigma^{\prime}-\left\{\left\langle q_{0}, p_{0}\right\rangle\right\}, \operatorname{pred}(\sigma) \subset \Sigma^{\prime}$

Note that the set of trace pairs $\Sigma_{T_{1}, T_{2}}$, satisfies the prefix_closed relation, and so does the singleton trace pair $\left\{\left\langle q_{0}, p_{0}\right\rangle\right\}$.

Lemma 1. Given two traces $T_{1}$ and $T_{2}$ of a transformed program $P^{*}$ with inputs that differ only on high security values, and with trace-pair set $\Sigma$, then exactly one of the following properties holds true for every $\sigma \in \Sigma$ (depending on the form of $\sigma)$ :

(a) if $\sigma=\left\langle q_{i}, p_{j}\right\rangle$ then $\forall x \in V a r$, $\operatorname{env}_{q_{i}}(x) \neq \operatorname{env}_{p_{j}}(x) \Rightarrow\left[\operatorname{env}_{q_{i}}(\mathcal{L}(x))=e n v_{p_{j}}(\mathcal{L}(x))=1\right]$

(b) if $\sigma=\left\langle q_{i},-\right\rangle$ then env $_{q_{i}}(\mathcal{L}(p c))=1$

(c) if $\sigma=\left\langle-, p_{j}\right\rangle$ then env $v_{p_{j}}(\mathcal{L}(p c))=1$

The intuition behind this lemma is as follows. Since the two traces are executing the same code with identical initial values except for one or more high security variables, any divergence in values of variables must be due either to a direct flow from one of the high security variables, or an indirect flow. In the case of a direct flow, the transformation rules for assignment will update the label variables so that the flow is noted by a true-valued label variable. In the case of an indirect flow, the two execution traces must diverge. At the first point of divergence, env $(\mathcal{L}(p c))$ will be set to true, and it will remain true until the point where the two traces converge (which might never happen).

Proof. By induction over the size of sets satisfying the prefix_closed relation.

Base: For sets of size one, the only element is $\left\langle q_{0}, p_{0}\right\rangle$, which is the initial state of every $\langle\Sigma, \triangleleft\rangle$ structure and is the start state, where $p c_{q_{0}}=p c_{p_{0}}$, and $e n v_{q_{0}}$ agrees with $e n v_{p_{0}}$ on all low security input values. For all the high security input values that may differ in the two traces, as all their label variables are initialized to 1 , branch (a) of lemma 1 is trivially satisfied.

Induction Hypothesis: Assume that for all sets $\Sigma^{\prime}$ of size $n$ that satisfy prefix_closed $\left(\Sigma^{\prime}\right)$, for all $\sigma \in \Sigma^{\prime}, \sigma$ satisfies Lemma 1 .

Step: pick any $\sigma \notin \Sigma^{\prime}$, such that all its immediate predecessors are in $\Sigma^{\prime}$ $\left(\operatorname{pred}(\sigma) \cap \Sigma^{\prime}=\operatorname{pred}(\sigma)\right) . \sigma$ either has the form (Case 1) $\left\langle q_{i}, p_{j}\right\rangle$, or (Case 2) $\left\langle q_{i},-\right\rangle$, or $($ Case 3$)\left\langle-, p_{j}\right\rangle$. 
Case 1: $\sigma=\left\langle q_{i}, p_{j}\right\rangle$. Consider $\operatorname{pred}(\sigma)$.

Either $\left(\right.$ Case 1.1) $\operatorname{pred}(\sigma)=\left\{\left\langle q_{i-1}, p_{j-1}\right\rangle\right\}$, or (Case 1.2) $\operatorname{pred}(\sigma)=\left\{\left\langle q_{i-1},-\right\rangle\right.$, $\left.\left\langle-, p_{j-1}\right\rangle\right\}$.

Case 1.1: $\sigma=\left\langle q_{i}, p_{j}\right\rangle$ and $\operatorname{pred}(\sigma)=\left\{\left\langle q_{i-1}, p_{j-1}\right\rangle\right\}$.

By the induction hypothesis, $\left\langle q_{i-1}, p_{j-1}\right\rangle$ satisfies Lemma 1(a). Consider the form of $s_{m} t_{q_{i-1}}\left(=s t m t_{p_{j-1}}\right)$. If $s t m t_{q_{i-1}}$ is not an assignment statement, then the $e n v$ is the same in both $\sigma$ and $\operatorname{pred}(\sigma)$, so $\sigma$ satisfies Lemma 1(a).

If stmt $_{q_{i-1}}$ is an assignment statement, then it must have the form $x:=e$; If $\operatorname{env}_{q_{i}}(x)=e n v_{p_{j}}(x)$, then $\sigma$ satisfies Lemma 1(a). If $e n v_{q_{i}}(x) \neq e n v_{p_{j}}(x)$, then we know that there was some $y \in e$ such that If $e n v_{q_{i-1}}(y) \neq e n v_{p_{j-1}}(y)$. By the induction hypothesis, env $v_{q_{i-1}}(\mathcal{L}(y))=e n v_{p_{j-1}}(\mathcal{L}(y))=1$. By the transformation rule for the assignment statement, we know that $\mathcal{L}(x)$ is assigned $\mathcal{L}(p c) \vee\left(\vee_{y \in e} \mathcal{L}(y)\right)$. This will result in $\operatorname{env}(\mathcal{L}(x))=1$ in both $q_{i-1}$ and $p_{j-1}$. In all cases, $\sigma$ satisfies Lemma 1(a).

Case 1.2: $\sigma=\left\langle q_{i}, p_{j}\right\rangle$. and $\operatorname{pred}(\sigma)=\left\{\left\langle q_{i-1},-\right\rangle,\left\langle-, p_{j-1}\right\rangle\right\}$.

In this case, stmt $t_{i}$ must be the exit from a while or if statement. Let $\left\langle q_{l}, p_{m}\right\rangle=$ $g c a\left(\left\langle q_{i-1},-\right\rangle,\left\langle-, p_{j-1}\right\rangle\right)$. By the induction hypothesis, $\left\langle q_{l}, p_{m}\right\rangle$ satisfies Lemma 1(a). Also, by the induction hypothesis, in each state along the path from $\left\langle q_{l}, p_{m}\right\rangle$ to $q_{i}$ satisfies Lemma 1(b), and each state along the path from $\left\langle q_{l}, p_{m}\right\rangle$ to to $p_{j}$ satisfies Lemma 1(c) (i.e., env $(\mathcal{L}(p c))$ is 1 along these paths). Because of the latter two facts, we show that for any variable $x$, env $(\mathcal{L}(x))$ can only go from 0 to 1 along either path (it can never become 0 from 1 ).

Pick any $x \in V a r$, by the induction hypothesis, if $e n v_{q_{l}}(x) \neq e n v_{p_{m}}(x)$, then $e n v_{q_{l}}(\mathcal{L}(x))=e n v_{p_{m}}(\mathcal{L}(x))=1$, and if $x$ is not assigned anywhere between $\left\langle q_{l}, p_{m}\right\rangle$ to $\left\langle q_{i}, p_{j}\right\rangle$, then the same value of $x$ and $\mathcal{L}(x)$ will propagate to $\left\langle q_{i}, p_{j}\right\rangle$, so $\left\langle q_{i}, p_{j}\right\rangle$ will satisfy env $v_{q_{i}}(x) \neq \operatorname{env}_{p_{j}}(x) \Rightarrow \operatorname{env}_{q_{i}}(\mathcal{L}(x))=e n v_{p_{j}}(\mathcal{L}(x))=1$. The same is true if $e n v_{q_{l}}(x)=e n v_{p_{m}}(x)$, and $x$ is assigned to along both paths (since env $(\mathcal{L}(x))$ will become 1 as env $(\mathcal{L}(p c))=1$ at the point of assignment until the merge).

The only remaining case is where $e n v_{q_{l}}(x) \neq e n v_{p_{m}}(x)$, and $x$ is assigned to along on one path (without loss of generality assume it is the path from $q_{l}$ to $\left.q_{i}\right)$, and $x$ is not assigned to along the other path $\left(p_{m}\right.$ to $\left.p_{j}\right)$. There are two ways this could happen: a) execution flow follows two different paths of an if or b) execution flow passes once through a while loop on $q_{l} \ldots q_{i}$ but not on $p_{m} \ldots p_{j}$.

In both these cases, there will be implicit flows from all the variables in the condition plus $\mathcal{L}(p c)$ to $\mathcal{L}(x)$ along the path where $x$ is not modified $\left(p_{m} \ldots p_{j}\right)$. Since, env $(\mathcal{L}(p c))=1$, env $(\mathcal{L}(x))$ will become one, (by the transformation rules for assignment) and $e n v_{p_{j}}(\mathcal{L}(x))=1$. The final result is that in $\left\langle q_{i}, p_{j}\right\rangle$, $e n v_{q_{i}}(x) \neq \operatorname{env}_{p_{j}}(x) \Rightarrow e n v_{q_{i}}(\mathcal{L}(x))=e n v_{p_{j}}(\mathcal{L}(x))=1$.

In every possible sub-case of case 1.2 , for any $x \in \operatorname{Var}, \operatorname{env}_{q_{i}}(x) \neq e n v_{p_{j}}(x) \Rightarrow$ $\operatorname{env}_{q_{i}}(\mathcal{L}(x))=\operatorname{env}_{p_{j}}(\mathcal{L}(x))=1$, hence $\left\langle q_{i}, p_{j}\right\rangle$ satisfies Lemma 1(a).

Case 2: $\sigma=\left\langle q_{i},-\right\rangle$.

Consider pred $(\sigma)$. Either (Case 2.1) $\operatorname{pred}(\sigma)=\left\{\left\langle q_{i-1},-\right\rangle\right\}$, or (Case 2.2) pred $(\sigma)=$ $\left\{\left\langle q_{i-1}, p_{j}\right\rangle\right\}$. 
Case 2.1: $\sigma=\left\langle q_{i},-\right\rangle$ and $\operatorname{pred}(\sigma)=\left\{\left\langle q_{i-1},-\right\rangle\right\}$.

By the induction hypothesis, $e n v_{q_{i-1}}(\mathcal{L}(p c))=1$. Consider If $q_{i-1}$ is not the exit from (i.e., last state in the execution of) an if or while, then $\operatorname{env}(\mathcal{L}(p c))$ is preserved in $q_{i}$, and $\sigma$ satisfies Lemma 1(b). If $q_{i-1}$ is the exit from an if or while, then stmt $t_{i-1}$ must be embedded within an enclosing if or while (otherwise, the second trace would reach the same extended program counter location and $\sigma$ would have the form $\left\langle q_{i}, p_{j+1}\right\rangle$. In the latter case, env $(\mathcal{L}(p c))$ will still be 1 when the if or while exits according to the transformation rules. So, in both cases, $\sigma$ satisfies Lemma 1(b).

Case 2.2: $\sigma=\left\langle q_{i},-\right\rangle$ and $\operatorname{pred}(\sigma)=\left\{\left\langle q_{i-1}, p_{j}\right\rangle\right\}$.

In this case, pred $(\sigma)$ must be the beginning of an if or while with two successors (due to trace divergence). That means that the expression $e$ used to determine the branch outcome evaluated differently in $q_{i-1}$ and $p_{j-1}$, which must be due to some set of variables $X$ whose values differ in $e n v_{q_{i-1}}$ and $e n v_{p_{j-1}}$. By the induction hypothesis, for each $x \in X, \mathcal{L}(x)$ evaluates to 1 in both $e n v_{q_{i-1}}$ and $e n v_{p_{j-1}}$. According to the transformation rules, $\mathcal{L}(p c)$ will be bound in $e n v_{q_{i}}$ to the result of or-ing the $\mathcal{L}(x)$ values together (along with some other values), so the result will be $e n v_{q_{i}}(\mathcal{L}(p c))=1$. Hence $\sigma$ satisfies Lemma 1(b).

Case 3: $\sigma=\left\langle-, p_{j}\right\rangle$.

By an argument symmetric to Case 2, $\sigma$ satisfies Lemma 1(c).

Conclusion: In all cases, Lemma 1 is satisfied. By induction, all $\sigma \in \Sigma$ reachable by traversing $\triangleleft$ from $\left\langle q_{0}, p_{0}\right\rangle$ satisfy Lemma 1 .

Theorem 2. The transformation $\rightarrow_{\Gamma, \Psi}$ respects non-interference, i.e., if the program $P$ has two traces that differ on assignments to low security variables (thus causing differing observable outputs) when only high security input values differ in the program, then the transformed program $P^{*}$ exits before such an assignment occurs.

Proof. Consider the differing assignment statement. Depending on whether the assignment to the low security variable happened in both traces, two cases are possible.

Case a) The assignment happens on both the traces with different values assigned to the low security variable $x$ (say). In this case, the trace pair is of the form $\left\langle q_{i}, p_{j}\right\rangle$ which satisfies Lemma 1(a), since the values assigned to $x$ are different, then $e n v_{q_{i}}(\mathcal{L}(x))=e n v_{p_{j}}(\mathcal{L}(x))=1$ before the assignment, and the policy_check procedure inserted before the assignment halts on inspecting this value of $\mathcal{L}(x)$.

Case b) The assignment happens in only one trace. In this case, the trace pair is of the form $\left\langle q_{i},-\right\rangle$ or $\left\langle-, p_{j}\right\rangle$. env $v_{i}(\mathcal{L}(p c))=e n v_{p_{j}}(\mathcal{L}(p c))=1$ by Lemma 1(b) ( or by Lemma 1(c)). Hence by the transformation rule for the assignment statement, for the variable $x$ that is assigned, env $v_{q_{i}}(\mathcal{L}(x))$ and $e n v_{p_{j}}(\mathcal{L}(x))$ will become 1. The policy_check procedure will halt the execution in a similar fashion. Hence the proof. 


\section{Practical Issues in Runtime Information Flow Enforcement}

Any approach for dealing with information flow must deal with several practical issues involved with general purpose programs. We discuss the key issues related to static analysis for the transformation, optimizations, variable aliasing, and information declassification in this section.

\subsection{Static Analysis for Transformation}

Our transformation is parameterized by a static analysis that can compute the set of variables $U p d(S)$ that are updated in a compound statement $S$. For statements that do not involve procedural calls, this analysis can simply collect all the LHS variables of assignments in the compound statement $S$ and return the results as $U p d(S)$. When procedural calls are involved, an inter-procedural side effect analysis is needed to compute $U p d(S)$. Many such analysis algorithms (e.g. [9]) are found in the literature.

The precision of the static analysis for computing update sets will affect the precision of runtime monitoring of the transformed program. When such a static analysis is imprecise and conservative, the variables that never get assigned in the statement $S$ may be included in $\operatorname{Upd}(S)$. Updating the label variable of such a variable with $\mathcal{L}(p c)$ can lead to false alarms on information leaks because the implicit flows from the branch condition in $S$ to the variable in question actually do not present in the program. We note that our approach is conservative yet sound in these cases and will not fail to detect the actual information leaks happened in the program.

\subsection{Optimizations}

The transformation presented in Section 2 was kept simple, avoiding all possible optimizations, for the sake of readability and clarity. In this section, we discuss techniques to optimize the performance of the run-time approach.

- Optimizations to transformation rules (if-then-else and while). We first note that the transformation for the if-then-else and while statements include updates to all the variables in the update set (denoted in the figure as $\left.U p d\left(S_{1}\right) \cup U p d\left(S_{2}\right)\right)$, Note that it adds updates to label variables after executing either branch. This is clearly redundant, as we need to only update the label variables corresponding to the branch not taken (to account for implicit flows). This simple optimization reduces the number of instructions added for tracking implicit flows directly to one half of the original number. A similar optimization can be done for the while statement to reduce the number of instructions needed to track implicit flows.

- Optimizations based on information type inference results. As noted earlier in the introduction, we note that our approach can be used to maintain 
safe information flows when the static type analysis checks do not declare the program as safe. We can leverage on the results of such as type analysis to further optimize the number of instructions added for runtime tracking. We can first use static information flow analysis/inference to propagate the sensitive types (only the high values) across the program. This propagation of sensitive types will result in partitioning the set of program variables into two pools: the sensitive pool, that denote potentially sensitive variables, and the non-sensitive pool, that denote variables that do not receive sensitive information. A key insight here is that variables from the non-sensitive pool do not ever receive sensitive information throughout their lifetime in any execution of the program. Hence runtime tracking is unnecessary for these variables. With this information, label variables are not added for these variables, and no updates are made (either for implicit or explicit flows) for these variables. This second step further reduces the number of instructions added to the original program. In addition, if the program counter label $\mathcal{L}(p c)$ is ever augmented with this label variable (if the original variable is used in a condition), then this update to $\mathcal{L}(p c)$ need not be performed at all. This will also mean that $\mathcal{L}(p c)$ does not require to be saved and restored before and after the body of this statement.

- Use of standard compiler optimizations for eliminating dead assignments to label variables. Modern compilers have several intraprocedural optimizations that are built-in. These assignments optimize the original program code. We note that when such optimizations happen, the same optimizations can also be performed on the corresponding label variables, thereby reducing the number of additional instructions to the original program. For instance, every dead assignment in the original program has a corresponding dead label variable assignment and this can be eliminated. This can be performed by a standard compiler that supports live variable analysis followed by dead assignment elimination of the program, resulting in code that is further optimized on label assignments. In a similar fashion, other compiler optimizations such as copy propagation, constant folding and loop optimizations can be applied to label variables as well, resulting in reducing the number of such label assignments.

Analysis of the number of additional instructions introduced due to the transformation. We now briefly discuss the additional number of instructions (label variable updates) introduced by the transformation. The transformation introduces label assignments for two cases (i) explicit flows and (ii) implicit flows. In the case of explicit flows, there is exactly one assignment for each assignment to a program variable. For the case of implicit flows, it may seem as if the number of added assignments is equal to the number of branch assignment statements in both branches. However, this is not true. The transformation adds only one assignment for every updated variable in the branch not taken. Hence the number of added label assignments is bounded by the number of unique variables updated in both branches. The effect of the above optimizations due to results 
from the type analysis and standard compiler optimizations reduces the number of such label variable assignments even further.

\subsection{Handling Aliasing}

An alias of a variable refers to the same storage location of the variable through a different name. Writes to the storage location through an alias variable will affect the accesses through all other aliases. Variable aliasing is common in modern program languages such as Java and $\mathrm{C}++$. An alias to a variable can be generated by creating a pointer or reference to the variable (e.g. by passing an object to another method in Java, or by using the reference operator \& in $\mathrm{C}++$ ).

In order to correctly track how the sensitive information flows in a program in the presence of aliasing, our transformation should ensure that the label variables of the aliased variables are also similarly aliased each other. However, it is rather difficult to synchronize such an aliasing relationship of label variables with that of program variables.

To solve this problem, we can use a pointer alias analysis (e.g. 3311]) to partition variables in a program into different sets, each of which contains only the variables that are aliased each other. We then assign one single label variable to each of such variable sets in the transformed program. This ensures that the label variable for each set of aliased variables gets updated properly for both explicit and implicit information flows into these variables.

\subsection{Declassification}

Declassification of information is required to downgrade sensitive information intentionally to a low security value, when dictated by certain situations. A classic example is a password program that accepts input (low) from a user, and compares it to the stored (high) password. Such comparison is bound to leak information, such leaks are generally considered acceptable for programs that have to deal with disclosure of such small amounts of sensitive information. Recent works in information flow analysis have looked at issues in safe uses of such declassifications 1917 .

Declassification can be introduced either as part of the original language through a special declassify operator, or as external specifications to the program. The declassify operator takes an expression as parameter. The use of an operator needs changes to the compiler framework for the language, as well as changes to existing programs, while making the intent explicit in the source code. The use of an external specification does not require both, but may have readability issues for someone reading the program. Regardless of how the declassification is specified, it is provided as input to our transformation framework, and associated static analysis mechanisms.

The process of tracking flows with the presence of declassification is straightforward. On the instance of a declassify operator, say, the label value arising out of the expression that is declassified is set to false. This has the net effect of 
downgrading, and no additional changes to the transformation rules are necessary. On the other hand, static analysis mechanism used may benefit from such specifications. As described earlier, they may possibly eliminate any variables assigned out of such declassified expressions from the update sets.

\section{Related Work}

Approaches addressing the information flow problem broadly fall into three categories: runtime analysis approaches, static analysis approaches, and theorem proving based approaches.

Runtime analysis approaches. Early work on protecting confidentiality of data involved the use of runtime monitoring. This was mainly started with the development of mandatory access control model in the context of multi-level security by Bell and LaPadula [5. Subsequent models, such as Fenton [13, followed this approach in the context of programs, in which the sensitivity of the output of a computation was calculated along with the computation. However, this approach fails to protect implicit flows due to paths that are not taken. Since then, most approaches started focusing on using static analysis techniques.

The scripting language Perl has a taint mode 32 that tracks data that arrives from untrusted sources (such as the network). Perl also supports implicit downgrading data from "tainted" to "untainted" through pattern matching. However, Perl does not track implicit flows. Recently, several taint mechanisms have appeared in the context of protecting system integrity [22 |8|26]6] Similar to Perl, none of these mechanisms handle implicit flows. The taint mechanism described in 34] has some limited support for implicit flow. However, there are no guarantees about non-interference properties.

Static analysis checks have been considered out of EM enforcement mechanisms 25. While EM-enforceable properties are those over traces, information flow properties are not EM- enforceable, as they are properties of trace sets [30. Hence, a purely runtime mechanism will not be able to enforce information flow policies. In this paper, we augment the execution trace with information about other possible executions that relate to the current execution. By doing this, we gain the ability to enforce information flow policies on programs.

Two other works use dynamic approaches in the context of the information flow analysis problem to address some of the limitations that were discussed in the introduction. The first is due to Zheng et al 35, which provides support for dynamically providing the values of labels for data items (such as a file whose access permission is not known). The second work by Tse et al 28, provides similar support for unknown principals (that are only available at runtime) that interact with the system. In both these works, the use of dynamic techniques is to expand the scope of the static analysis based policy enforcement mechanism. However, they still do not support programs that may occasionally leak information such as the crash example discussed in the introduction.

Guernic and Jensen [15] have independently developed a similar approach that provides non-interferences guarantees based on a single run of a program. They 
present their approach through an operational semantics, but do not provide a proof. Our work 29] precedes their work.

Static analysis approaches. Various static analysis based approaches have been used for information flow analysis. (We only discuss most representative work here, a detailed survey article on various language based techniques for tracking information flow is available 24]).

Denning's approach 12 based on program certification was the first work that used an augmented compiler to track information flows. Andrews and Reitman [1] used an extended axiomatic logic with the secure flow certification of Denning. The work of Volpano et al [31, is based on the use of type-analysis for detecting information flows, and the approach is provably secure in handling implicit flows.

Myers presents a decentralized label model [21] for information flow where the owner specifies the access rights of data owned by her and the language, Jif, uses a type-analysis combined with runtime checks to detect illegal flows [20]. This is the first work that addressed the problem of information flow in a real programming language realm. Flow Caml 23, developed by Simonet and Pottier, is also a high-level, realistic programming language aiming at support information-flow controls. More recently, a certifying compiler for information flow policies was described in Barthe 4. Banerjee and Naumann 2] connect information-flow policies and stack inspection (an access control mechanism) for static checking of information flow policies in a Java-like language. Mclean [18 presents a specification of a program using trace semantics and develops a systematic theory to enable reasoning about non-interference for such specifications.

As we pointed out in the introduction, static analysis based approaches have drawbacks that may result in rejection of safe programs. Information leaks in certain unusual cases are common in many useful programs. Static analysis based approaches will reject such programs, while our runtime analysis based approach will permit the execution of such programs and halt them only when the actual information leak happens.

Theorem proving based approaches. Precise characterization of information flow is inherently an undecidable problem. Static analysis based mechanisms guarantee termination by providing a sound solution to the problem, with the possibility of rejecting safe programs. The approach taken originally in Joshi et al [16, and more recently by Darvas et al [10] and Barthe et al 3] use theorem proving as a technique to make improve the precision of static analysis. This is done by characterizing information flow as a safety problem (using a technique called self-composition, summarized in a formulation by [27]) and using theorem proving technology to certify programs as safe. Theorem proving certainly has the ability to provide more precise results. However, in the case of all theorem proving techniques, there is a risk of non-termination as exemplified by a simple example discussed in [27]. Static analysis mechanisms, by definition, always terminate. Our focus in this work is to occupy a position that is midway between static analysis and theorem proving. We try to support execution of programs that are rejected by static analysis by improving precision, and also by providing a transformation approach that always terminates. 


\section{Conclusion}

As we have shown, runtime detection of implicit information flows are possible when information from a simple static analysis are combined with program instrumentation which performs runtime flow tracking. Furthermore, we have established the soundness of such an approach with respect to the non-interference property. The contributions of this paper is mainly theoretical in nature. In future work, we plan to implement this technique and evaluate its effectiveness and performance in practice.

Although the presented approach ensures confidentiality of secure data, it can easily be changed to ensure integrity as well. In this case, the objective is to prevent the flow of information from untrusted variables to trusted ones. The primary change that is required is in the initial labeling of variables; otherwise, the propagation rules for labels are similar.

\section{References}

1. G. R. Andrews and R. P. Reitman. An axiomatic approach to information flow in programs. ACM Transactions on Programming Languages and Systems (TOPLAS), 2(1):56-75, 1980.

2. A. Banerjee and D. A. Naumann. Using access control for secure information flow in a java-like language. In Proc. IEEE Computer Security Foundations Workshop, 2003.

3. G. Barthe, P. D'Argenio, and T. Rezk. Secure information flow by self-composition. In Proc. IEEE Computer Security Foundations Workshop, 2004.

4. G. Barthe, T. Rezk, and D. Naumann. Deriving an information flow checker and certifying compiler for java. In IEEE Symposium on Security and Privacy, 2006.

5. D. E. Bell and L. J. LaPadula. Secure computer systems: Mathematical foundations. Technical Report MTR-2547, Vol. 1, MITRE Corp., 1973.

6. Y. Beres and C. Dalton. Dynamic label binding at run-time. In New Security Paradigms Workshop, 2003.

7. P. Broadwell, M. Harren, and N. Sastry. Scrash: A system for generating security crash information. In USENIX Security Symposium, 2003.

8. S. Chen, J. Xu, N. Nakka, Z. Kalbarczyk, and R. K. Iyer. Defeating memory corruption attacks via pointer taintedness detection. In IEEE International Conference on Dependable Systems and Networks (DSN), 2005.

9. K. D. Cooper and K. Kennedy. Interprocedural side-effect analysis in linear time. In Programming Languages Design and Implementation (PLDI), 1988.

10. A. Darvas, R. Hähnle, and D. Sands. A theorem proving approach to analysis of secure information flow. In D. Hutter and M. Ullmann, editors, Proc. 2nd International Conference on Security in Pervasive Computing, volume 3450 of LNCS, pages 193-209. Springer-Verlag, 2005.

11. M. Das. Unification-based pointer analysis with directional assignments. In Programming Languages Design and Implementation (PLDI), 2000.

12. D. E. Denning and P. J. Denning. Certification of programs for secure information flow. Comm. of the ACM, 20(7):504-513, 1977.

13. J. S. Fenton. Memoryless subsystems. Computing J., 17(2):143-147, 1974. 
14. J. A. Goguen and J. Meseguer. Security policies and security models. In IEEE Symposium on Security and Privacy, pages 11-20, 1982.

15. G. L. Guernic and T. Jensen. Monitoring information flow. In Workshop on Foundations of Computer Security, 2005.

16. R. Joshi and K. R. M. Leino. A semantic approach to secure information flow. Science of Computer Programming, 37(1-3):113-138, 2000.

17. P. Li and S. Zdancewic. Downgrading policies and relaxed noninterference. In ACM Symposium on Principles of Programming Languages (POPL), 2005.

18. J. McLean. Proving noninterference and functional correctness using traces. Journal of Computer Security, 1(1), 1992.

19. A. Myers, A. Sabelfeld, and S. Zdancewic. Enforcing robust declassification. In Proc. IEEE Computer Security Foundations Workshop, 2004.

20. A. C. Myers. JFlow: Practical mostly-static information flow control. In ACM Symposium on Principles of Programming Languages (POPL), pages 228-241, 1999.

21. A. C. Myers and B. Liskov. Complete, safe information flow with decentralized labels. In IEEE Symposium on Security and Privacy, pages 186-197, 1998.

22. J. Newsome and D. Song. Dynamic taint analysis for automatic detection, analysis, and signature generation of exploits on commodity software. In Network and Distributed System Security Symposium (NDSS), 2005.

23. F. Pottier and V. Simonet. Information flow inference for ml. In ACM Symposium on Principles of Programming Languages (POPL), 2002.

24. A. Sabelfeld and A. C. Myers. Language-based information-flow security. IEEE J. Selected Areas in Communications, 21(1), 2003.

25. F. B. Schneider. Enforceable security policies. ACM Transactions on Information and System Security (TISSEC), 3(1), 2001.

26. G. E. Suh, J. W. Lee, D. Zhang, and S. Devadas. Secure program execution via dynamic information flow tracking. In International Conference on Architectural Support for Programming Languages and Operating Systems, pages 85-96, 2004.

27. T. Terauchi and A. Aiken. Secure information flow as a safety problem. In Static Analysis Symposium (SAS), 2005.

28. S. Tse and S. Zdancewic. Run-time principals in information-flow type systems. In IEEE Symposium on Security and Privacy., 2004.

29. V. N. Venkatakrishnan, D. C. DuVarney, W. Xu, and R. Sekar. A program transformation technique for enforcement of information flow properties. Technical Report SECLAB-04-01, Department of Computer Science, Stony Brook University, 2004.

30. D. Volpano. Safety versus secrecy. In Static Analysis Symposium (SAS), volume 1694 of Lecture Notes in Computer Science, pages 303-311, 1999.

31. D. Volpano, G. Smith, and C. Irvine. A sound type system for secure flow analysis. Journal of Computer Security (JCS), 4(3):167-187, 1996.

32. L. Wall, T. Christiansen, and R. Schwartz. Programming Perl. O'Reilly, 1996.

33. J. Whaley and M. S. Lam. Cloning-based context-sensitive pointer alias analysis using binary decision diagrams. In Programming Languages Design and Implementation (PLDI), 2004.

34. W. Xu, S. Bhatkar, and R. Sekar. Taint-enhanced policy enforcement: A practical approach to defeat a wide range of attacks. In USENIX Security Symposium, 2006.

35. L. Zheng and A. Myers. Dynamic security labels and noninterference. In Workshop on Formal Aspects in Security and Trust (FAST), 2004. 